\title{
Management of right coronary artery fistula caused by nail gun trauma
}

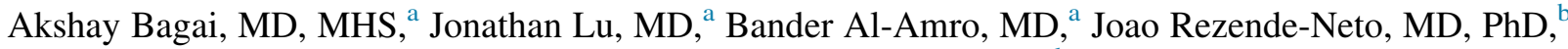 \\ Vikramaditya Prabhudesai, MBBS, MS, ${ }^{\mathrm{c}}$ Alun D. Ackery, MD, MSc, ${ }^{\mathrm{d}}$ and Bobby Yanagawa, MD, PhD, \\ Toronto, Ontario, Canada
}

\footnotetext{
From the Divisions of ${ }^{\mathrm{a}}$ Cardiology, ${ }^{\mathrm{d}}$ Emergency Medicine, and ${ }^{\mathrm{e}}$ Cardiac Surgery, Terrence Donnelly Heart Center, and Divisions of ${ }^{b}$ General Surgery and ${ }^{c}$ Radiology, St Michael's Hospital, University of Toronto, Toronto, Ontario, Canada.

Disclosures: Authors have nothing to disclose with regard to commercial support.

Received for publication June 9, 2016; revisions received July 14, 2016; accepted for publication July 17, 2016; available ahead of print Oct 5, 2016.

Address for reprints: Bobby Yanagawa, MD, PhD, Division of Cardiac Surgery, St Michael's Hospital, 30 Bond St, 8th Floor, Bond Wing, Toronto, Ontario M5B 1W8, Canada (E-mail: yanagawab@smh.ca).

J Thorac Cardiovasc Surg 2016;152:e139-41

$0022-5223 / \$ 36.00$

Copyright (C) 2016 by The American Association for Thoracic Surgery

http://dx.doi.org/10.1016/j.jtcvs.2016.07.086
}

Video clip is available online.

A 42-year-old carpenter, who was previously healthy, shot a nail into his chest with a pneumatic gun. He was brought by air ambulance to the hospital with transient inferior STsegment elevation and lateral ST-segment depression, but he was in a stable condition. A chest radiograph demonstrated a large nail with the tip in the region of the lower right atrium (Figure 1, $A$ ). Thoracic computed tomography (CT) showed the nail passing through the anterior wall of the right ventricular outflow tract (RVOT) and into the right atrioventricular groove (Figure 1, $B$ and $C$ ). The nail transected the proximal right coronary artery (RCA) where an $11 \times 6 \mathrm{~mm}$ pseudoaneurysm communicated with a 20-mm-long laceration tract that extended into the right

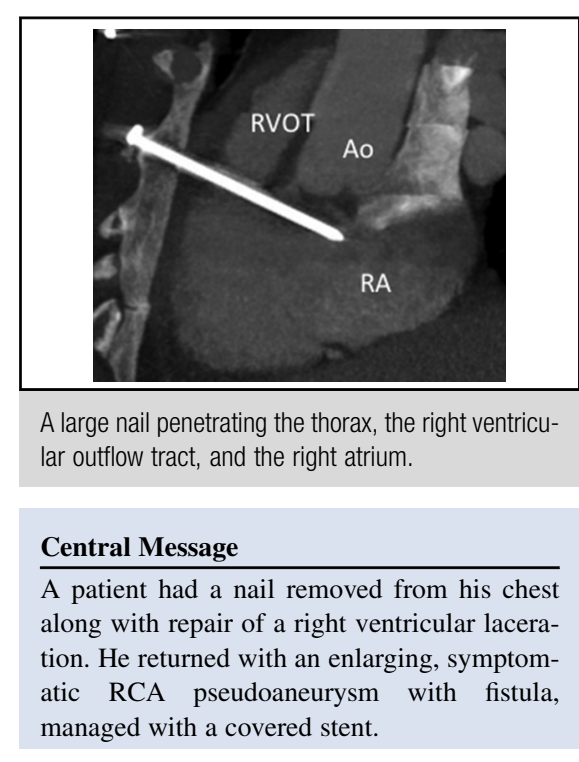

See Editorial Commentary page e143.

atrium just above the tricuspid annulus. There was a small hemopericardium with no evidence of tamponade.

The patient was brought to the operating room, prepped, and draped. The nail was removed under transesophageal echocardiography (TEE) guidance. Within 10 minutes, he developed a hemopericardium prompting sternotomy and direct repair of a right ventricular (RV) free wall laceration.
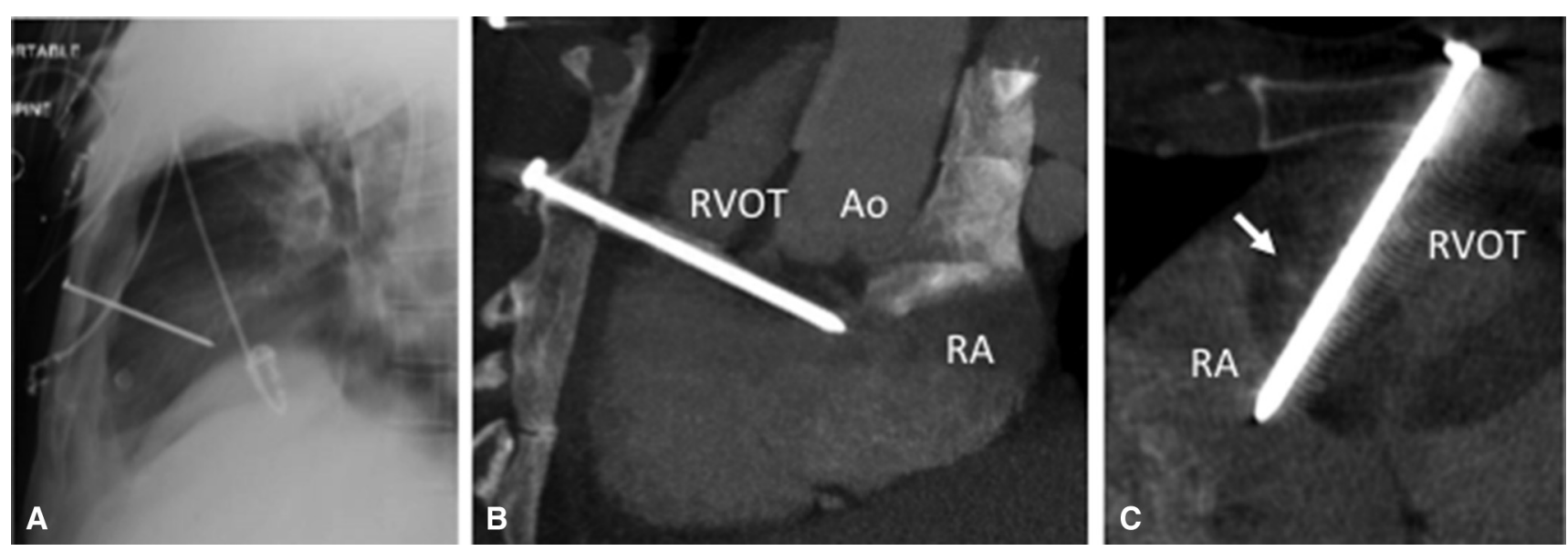

FIGURE 1. A lateral chest radiograph showing the nail penetrating the thorax (A). Computed tomography demonstrates the position relative to the nail to right ventricular outflow tract $(R V O T)$, right atrium $(R A)$, and right coronary artery (arrow; B and C). Ao, Aorta. 

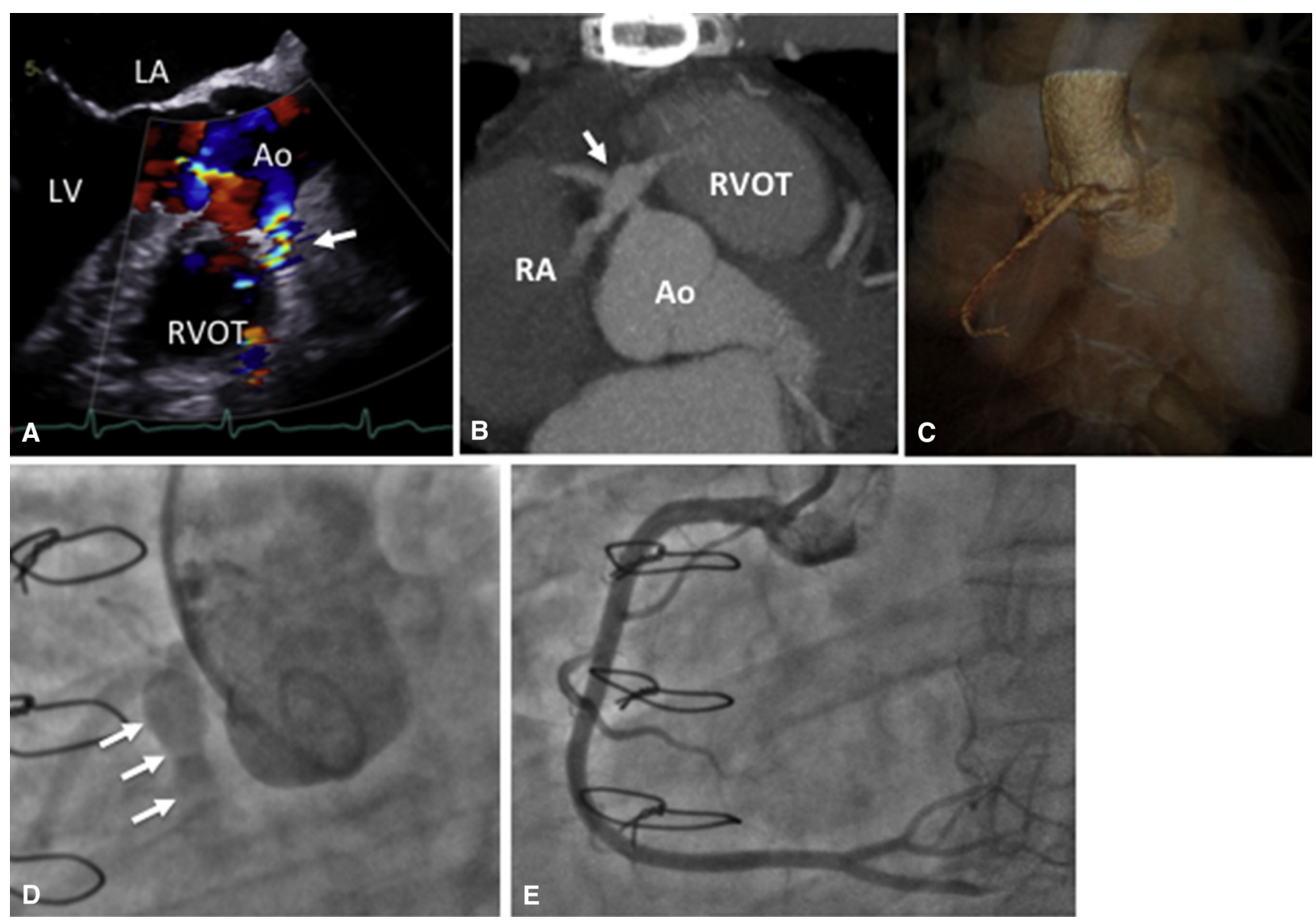

FIGURE 2. After surgical removal of the nail and repair of the right ventricular, a right coronaty artery-right ventricular outflow tract (RVOT) fistula (arrow) is shown by color flow Doppler on transesophageal echocardiography (A). A pseudoaneurysm of the right coronary artery (arrows) with fistula into the right atrium $(R A)$ and RVOT is also demonstrated by computed tomography (B and C). Angiographically, a lobulated pseudoaneurysm (arrows) is seen in the proximal right coronary with contrast extravasation in the right-sided chambers (D) and successful deployment of a covered stent (E). $L A$, Left atrium; $A o$, aorta; $L V$, left ventricle.

A small stable hematoma in the right atrioventricular groove was untreated and no additional injuries were noted. Predischarge transthoracic echocardiography (TTE) demonstrated a severely hypokinetic RV free wall. He recovered well and was discharged on postoperative day 5.

The patient sought medical attention 10 days after discharge for symptoms of night sweats and rigors. Cardiac auscultation revealed a new continuous murmur with systolic and diastolic components. TTE demonstrated a late-systolic and diastolic flow in the RVOT. There was no valvular vegetation, no leukocytosis, and blood cultures were negative. TEE demonstrated a dilated RCA ostium, with prominent systolic and diastolic color flow (peak velocity $3.6 \mathrm{~m} / \mathrm{s}$ ) in continuity with the color flow in the RVOT suspicious for a RCA to RVOT fistula (Figure 2, $A$; Video 1). Gated thoracic CT confirmed the suspected fistulous connection. There was a lobulated pseudoaneurysm beyond the origin of the RCA with connection to the right atrium and RVOT $(13 \times 11 \mathrm{~mm})$, which had increased in size (Figure 2, $B$ and $C$ ). Cardiac catheterization showed the following: right atrial pressure, $3 \mathrm{~mm} \mathrm{Hg}$; RV systolic pressure, $24 \mathrm{~mm} \mathrm{Hg}$; pulmonary arterial pressure, 26/7 mm Hg; and pulmonary capillary wedge pressure,

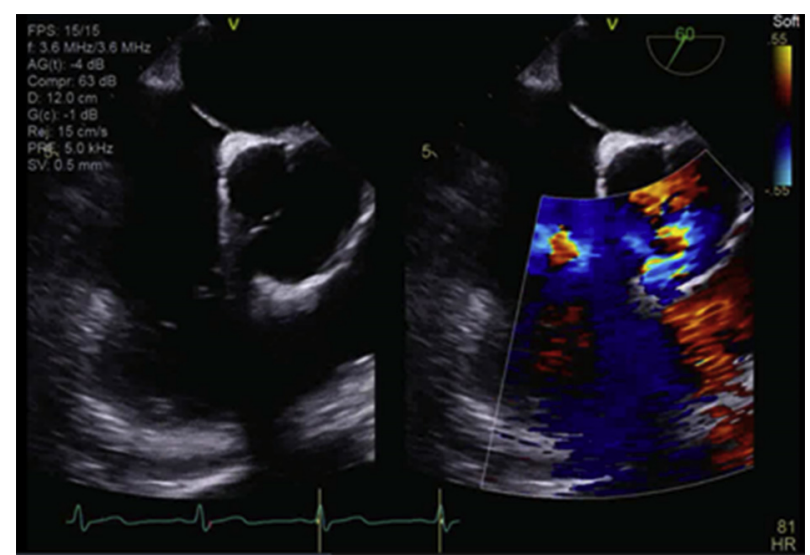

VIDEO 1. Right coronaty artery-right ventricular outflow tract and right atrium fistula is shown by color flow Doppler on transesophageal echocardiography. Video available at: http://www.jtcvsonline.org/article/ S0022-5223(16)31133-3/addons. 


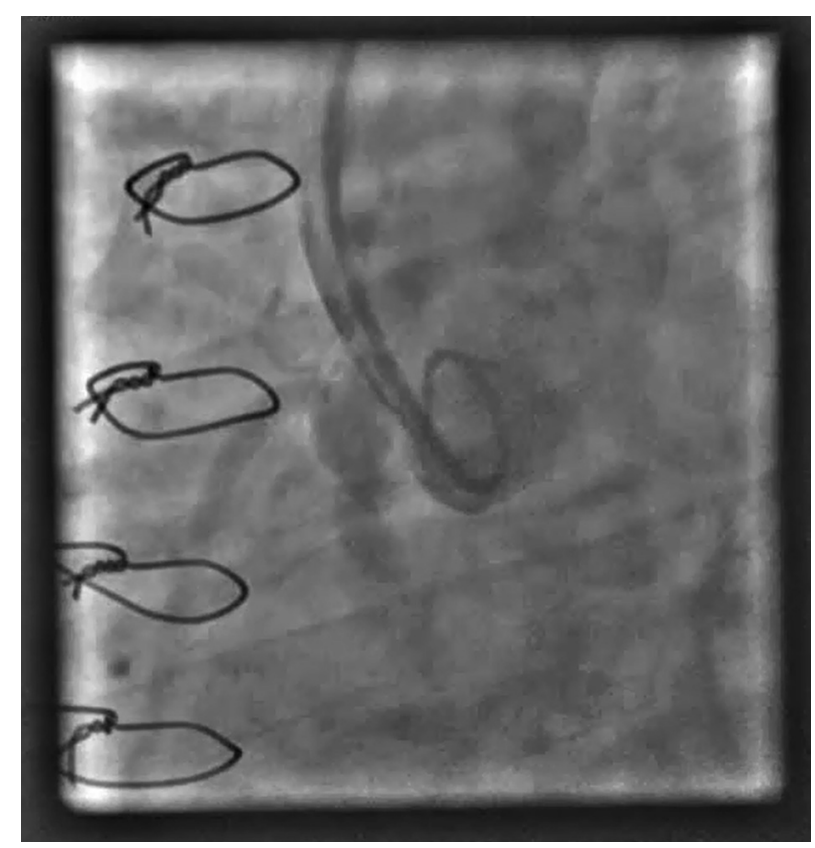

VIDEO 2. Aortic angiogram demonstrating a large pseudoaneurysm in the proximal right coronaty artery with contrast extravasation into right-sided cardiac chambers. Video available at: http://www.jtcvsonline. org/article/S0022-5223(16)31133-3/addons.

$7 \mathrm{~mm} \mathrm{Hg}$. There was no significant step up in oxygen saturation between the mid right atrium and RVOT. The calculated shunt fraction (Qp/Qs) was 1.2:1. An aortic root angiogram demonstrated a large, wide-neck pseudoaneurysm in the proximal RCA with large volume contrast extravasation into the right-sided cardiac chambers (Figure 2, D; Video 2). An exercise treadmill stress test was performed to assess for coronary steal. The patient exercised for 6 minutes on the Bruce protocol attaining a peak heart rate of $146 \mathrm{bpm}$ ( $82 \%$ of age-predicted maximum). He developed symptoms of dyspnea, chest pain, and presyncope with a drop in systolic blood pressure from 130 to $90 \mathrm{~mm} \mathrm{Hg}$. There were no significant ST-segment changes.

The decision was made to treat the fistulous connection using a covered stent. The RCA was non-selectively cannulated with an AR1 guiding catheter and successfully wired. The reference RCA diameter was $4.5 \mathrm{~mm}$ distal and $4.7 \mathrm{~mm}$ proximal to the pseudoaneurysm on intravascular ultrasound imaging. A $5.0 \times 20 \mathrm{~mm}$ Papyrus-covered stent (BIOTRONIK, Berlin, Germany) was deployed across the fistulous connection with $2 \mathrm{~mm}$ of stent protruding into the aorta. There was complete closure of the fistula coupled with thrombolysis in myocardial infarction grade 3 flow in the distal RCA (Figure 2, E; Video 3). The patient was discharged on lifelong aspirin and clopidogrel for 1 year. One month after fistula closure, he was asymptomatic without evidence of a fistulous connection.

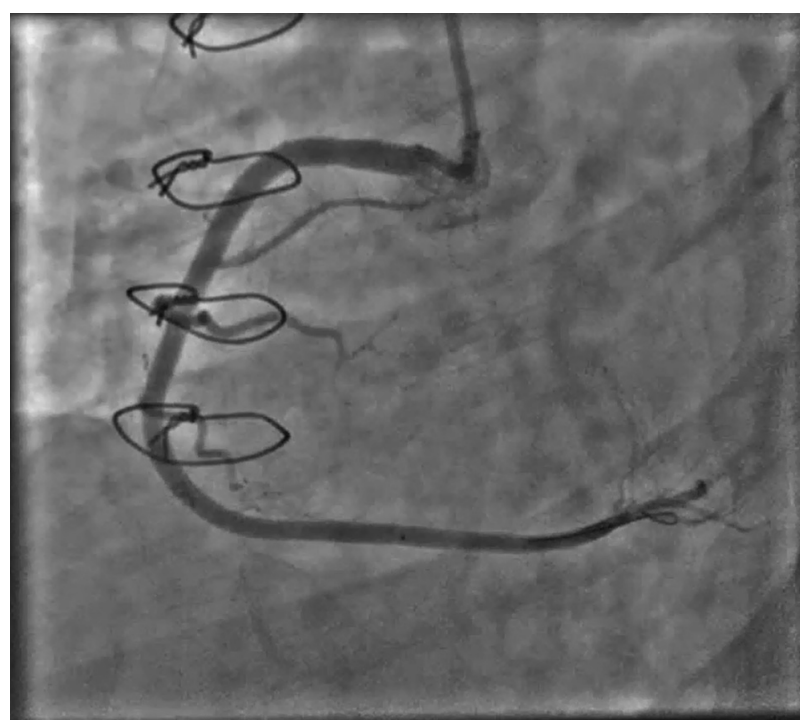

VIDEO 3. A selective right coronaty artery (RCA) angiogram after deployment of the Papyrus-covered stent (BIOTRONIK) in the proximal RCA demonstrating cessation of fistulous flow. Video available at: http:// www.jtcvsonline.org/article/S0022-5223(16)31133-3/addons.

\section{DISCUSSION}

Traumatic coronary artery fistulas resulting from penetrating chest injuries are rare. Patients may be asymptomatic at the time of initial injury only to develop a murmur or symptoms later such as congestive heart failure, pulmonary hypertension, or endocarditis. ${ }^{1,2}$ Intervention should be considered in most if not all patients, particularly in those who are symptomatic. Treatment options include (1) surgical correction with ligation of the coronary artery proximal and distal to the fistula ${ }^{3}$ and bypass grafting of the distal vessel, (2) percutaneous closure either with coil embolization, or (3) a covered stent. ${ }^{4}$ Bypass would have exposed this young patient to the risk of graft failure. Coil embolization was not ideal given the wide neck of the pseudoaneurysm and the risk of coil migration and thrombosis of the RCA. We used a Papyrus-covered stent, usually reserved for the treatment of coronary perforations, which instantaneously closed the fistulous flow. We plan to treat future in-stent restenosis with drug-eluting stent using percutaneous coronary intervention and then bypass as necessary. We believe covered coronary stents are an effective alternative strategy for closure of traumatic coronary artery fistulas.

\section{References}

1. Thandroyen FT, Matisonn RE. Penetrating thoracic trauma producing cardiac shunts. J Thorac Cardiovasc Surg. 1981;81:569-73.

2. Liberthson RR, Sagar K, Berkoben JP, Weintraub RM, Levine FH. Congenita coronary arteriovenous fistula. Report of 13 patients, review of the literature and delineation of management. Circulation. 1979;59:849.

3. Cheung DL, Au WK, Cheung HH, Chiu CS, Lee WT. Coronary artery fistulas: long-term results of surgical correction. Ann Thorac Surg. 2001;71:190-5.

4. Behera SK, Danon S, Levi DS, Moore JW. Transcatheter closure of coronary artery fistulae using the Amplatzer Duct Occluder. Catheter Cardiovasc Interv. 2006; $68: 242-8$ 